\title{
Toward Modeling of Cable-Harnessed Structures: Cable Damping Experiments
}

\author{
Kaitlin S. Spak ${ }^{1}$ \\ Virginia Polytechnic Institute and State University, Blacksburg, VA 24060 \\ Gregory S. Agnes ${ }^{2}$ \\ Jet Propulsion Laboratory, California Institute of Technology, Pasadena, CA 91011 \\ and \\ Daniel J. Inman ${ }^{3}$ \\ University of Michigan, Ann Arbor, 48109
}

\begin{abstract}
In order to develop models for space flight cables, the factors that affect the dynamic response of the cables must be determined. Toward this goal, this work presents the results from a set of experimental tests on $1 \times 18$ helically twisted cables made of 26 AWG insulated wire. The excitation method, tension in the cable, zip tie attachment method, and length and tension of the excitation string were varied for a single section of cable. In addition, five sections of cable were tested to investigate the variability within a single type of cable. The variability between the cable sections was as great as the variation due to any single test set up alteration, which indicates that a statistical approach may be necessary for cable characterization. Neither the excitation method nor length or tension in the excitation string afected the cable response significantly. Frequency response did change with changes in cable tension, as well as zip tie tightness. Thus, for best repeatability, cable tension and zip tie tightness should be controlled.
\end{abstract}

\section{Nomenclature}

$\begin{array}{ll}A & =\text { area of cable cross-section } \\ E & =\text { elastic modulus of the cable } \\ G & =\text { shear modulus } \\ I & =\text { area moment of inertia } \\ T & =\text { axial tension of the cable } \\ \rho & =\text { cable density }\end{array}$

\section{Introduction}

$\mathrm{D}$ UE to advances in material science and computing capabilities, space structures are becoming lighter in weight and contain more complex wiring than ever before. As the spacecraft mass decreases and the number of cable harnesses increases, the mass ratio of cable to spacecraft increases, and the added cables can significantly affect the dynamic response of the structure. Specifically, the added cables behave as a structural mass, and may contribute additional un-quantified damping to the structure. Being able to characterize, measure, and ultimately predict this additional damping could lead to greater spacecraft stability, more accurate modal testing and elimination of redesign after cable dressing, and a better understanding of damping mechanisms in general. A first step in this process is the understanding of the dynamic response and inherent damping of space flight cables themselves, as well as the calculation of cable parameters for use in predictive models. This work presents results for the variations in experimentally determined frequency response functions for a helically twisted $1 \times 18$ cable strand. From this data, cable properties and damping information can be eventually determined for model inputs. However, the primary

\footnotetext{
${ }^{1}$ PhD Candidate, Mechanical Engineering Dept., 1989 Sinaloa Ave., Altadena, CA 91001, AIAA Student Member.

${ }^{2}$ R\&D Engineer, 355L, 126-107, AIAA Associate Fellow.

${ }^{3}$ Department Chair, Aerospace Engineering Dept., 3064 FXB, AIAA Fellow.
} 
focus of this work is the identification of the effects of different test set up parameters on the frequency response functions of the cable.

\section{Background}

Research conducted by the Air Force Research Laboratory Space Vehicles Directorate shows that including cables as a lumped, non-structural mass is no longer adequate for accurate modeling. However, before modeling the cabled structure as a whole, there must be a greater understanding of the damping properties inherent in space cables, and the cable properties must be determined for modeling and predictive purposes. Thus, background research on cables and cable modeling was investigated. Most cable models fall into one of three categories: thinrod models, in which each wire of the cable is modeled as a thin rod, beam models, in which the cable as a whole is modeled as a beam, and semi-continuous models, in which each layer of wires in the cable is modeled as a continuous cylinder. To investigate frequency response and damping, the beam models provide the most straightforward modeling approach and have been successfully used in recent research. Goodding, Ardelean, Babuska, Robertson, and Lane developed cable test methods and were able to model simple twisted-pair cables through a combination of shear beam theory and experiments [1]. However, only one construction form of cable (twisted pairs bundled and stitched) was investigated, and the researchers note that there was significant build-to-build variability. Cable modal damping was roughly determined as a constant modal percentage.

Other cable damping models have been suggested recently, but have not ventured out of the viscous realm. Kauffman, Lesieutre and Babuska proposed a rotational viscous model that improved upon motion- or strain-based viscous damping models to quantify cable damping [2]. Without specific application to cables, hysteretic damping models for beams have been developed $[3,4]$ which may be a more accurate way to model cable damping. For this approach, cable properties must be measured to create equivalent $E, I$, and $A$ values, and hysteretic damping coefficients must be estimated or determined. It appears that there is more work to be done for both the experimental and modeling aspects of cable damping.

\section{Objective}

There is not yet a model that can reliably predict the frequency response changes of a structure caused by the addition of cable harnesses. The ultimate goal of this research will be to create a model that can quantify the damping added to a structure due to cable harnesses. However, before a model can be developed, the physical attributes that need to be included in the model must be determined. Therefore, the short term goal and scope of this paper is to determine what variables in the cable test set up affect the dynamic response of space flight cables. As shown in Fig. 1, a helically twisted $1 \times 18$ cable made of shielded and insulated 26AWG twisted wire pairs was excited and the frequency response function measured as different aspects of the test set up were varied. This paper will present the experimental results obtained.

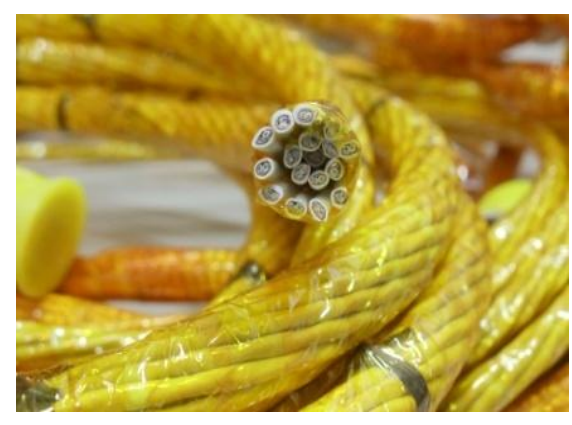

Figure 1. Helically twisted 1x18 space flight cable used for experimental testing.

\section{Experimental Set Up and Procedure}

The eventual goal of this work is to run experiments to quantify cable damping, and then compare that experimental data to as-yet unpublished models created by the author to incorporate hysteretic damping, cable

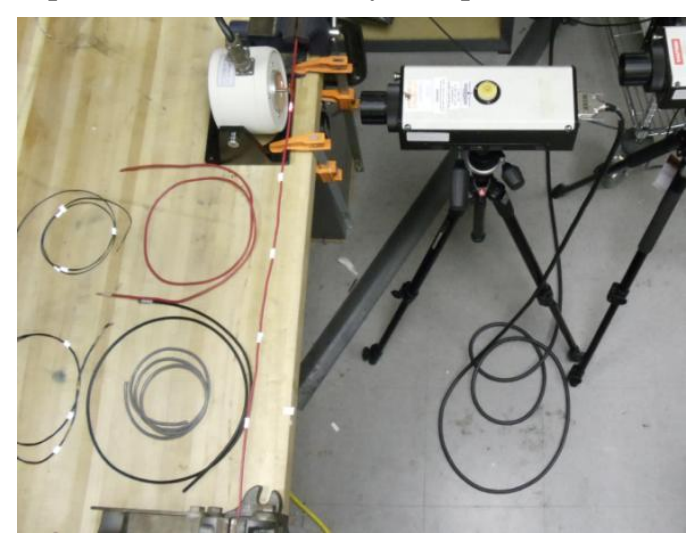

Figure 2. Testing of common electrical cables with clamped end conditions. tension, and variable bending stiffness. Preliminary experiments for cables and cabled beams were run at CIMSS Lab at Virginia Polytechnic Institute and State University, as shown in Fig. 2. From this preliminary work, the idea was extended to analyze space flight cables in a similar manner.

The space flight cable experiments presented here were performed at Jet Propulsion Laboratory in a controlled and isolated environment to eliminate the low-frequency noise that plagued the experiments run previously. A scanning laser vibrometer was used to gather data over the entire cable, and a tensioned string was used as the attachment point from the shaker to the cable. The shaker was suspended by long chains so that vibration from the shaker would not travel through the inertial table to the cable test fixture. A load cell attached to the cable

\section{2}

American Institute of Aeronautics and Astronautics 
measured the input force from the shaker. Figure 3 shows the test set up for flight-suitable cables, and Fig. 4 gives a closer view of the shaker connection through the load cell and the cable attachment point with a zip tie and TC 105 tab. This mounting method was used both because it is similar to a pinned condition for modeling purposes, and because securing cables to TC 105 tabs with zip ties is common practice on space structures, and therefore has real application value as well.

The natural frequencies of the suspended shaker and supporting structures were measured to ensure that they did not interact with the modes of interest. Additional pinned constraints were set at 8 " above and below the 10 " test section to provide buffer zones that served to both mitigate end effects and mimic the reality of a cable harnessed structure. An additional laser vibrometer was set up to measure transverse frequency responses. Cables were always fastened into the test fixture in the same way, with the inner side of the natural cable curve facing the shaker. Response was measured at the driving point for both the excitation plane and the perpendicular plane. Preliminary tests were run on a variety of cables to determine frequencies of interest and what parameters might need to be varied. From these tests, a "standard" test run was developed, which included the following controlled characteristics:

- $\quad 0.254 \mathrm{~m}$ test section length secured by zip ties tightened to setting 5 on zip tie gun (tight)

- $\quad 8.89 \mathrm{~N}$ of tension in the cable (hose clamp secured to cable distributed weight evenly)

- White noise excitation applied at 0.3 Volts

- Excitation applied at $8.3 \mathrm{~cm}$ from bottom of test section via $0.24 \mathrm{~m}$ tensioned string at medium DC offset

- Static cable displacement due to excitation string tension less than $0.6 \mathrm{~mm}$

- Low pass $5 \mathrm{kHz}$ filter and Hanning window applied, 30 averages per test run On each day of testing, the cable response was also scanned once at intervals of 0.9 centimeters, encompassing the entire test section, to visualize the mode shapes and ensure that the cable transverse modes were identified correctly.

The various tests deviated from the standard run only by the variable of interest for each test. The varied parameters were the type of excitation, the tension and length of the string used for the excitation connection, the tension in the cable, and the type and tightness of the zip ties used at the test section ends. In addition, the cable of interest was cut into five equal length sections and the frequency response of the different sections were compared in standard runs with interesting results.

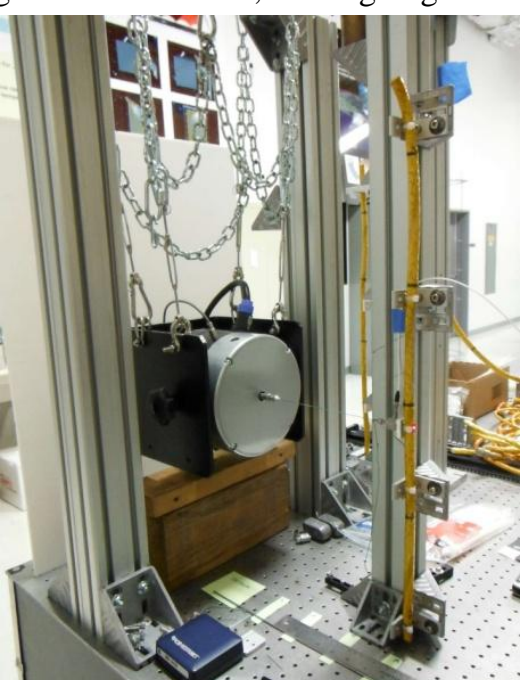

Figure 3. Testing of space flight cable with pinned end conditions, suspended shaker, and load cell force input in controllable environment.

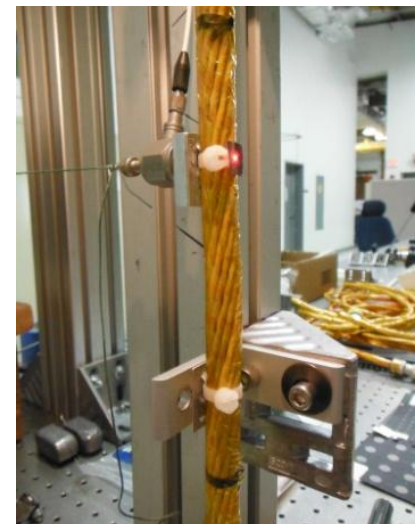

Figure 4. Cable attachment point and driving point.

\section{Experimental Tests and Results}

Initial investigation began with the test fixture and excitation connection. A slender narrow solid metal stinger was rejected in favor of a tensioned string after determining that the response was basically equivalent, but the string did not support a moment or lateral force, so only transverse force was received from the shaker.

Preliminary experiments on cables of varying sizes showed that some method was needed to ensure that the cable was not experiencing significant bending due to displacement at the excitation point since initial research showed that cable bending stiffness is related to cable curvature. A laser displacement system was set up to make sure that once the shaker string was attached and tensioned, the cable had not been bent by more than $1 \mathrm{~mm}$. Large diameter cables (greater than $1 \mathrm{~cm}$ in diameter) generally had a static displacement of about $0.6 \mathrm{~mm}$ with smaller cables being more flexible and thus more susceptible to bending due to the tensioned wire. For later testing of cables of varying sizes, care should be taken to prevent smaller cables from being bent by the shaker connection.

All cables were wrapped with Kapton tape, but one in our preliminary tests was tied with twist ties every $16 \mathrm{~cm}$. This cable appeared to maintain its responses better than non-tied cables, so this type of cable was chosen for the variation testing.

A full set of tests was performed each day on cable section A over the course of two weeks; this full set included comparisons of excitation methods, excitation string length and tension, cable tension, and zip tie attachment. The responses of sections $\mathrm{B}, \mathrm{C}, \mathrm{D}$, and $\mathrm{E}$ were also measured each day, and were run five times each at the conclusion of 
the test to provide additional data for statistical analysis. In general, the first natural frequency was around $55 \mathrm{~Hz}$ and the second natural frequency was around $180 \mathrm{~Hz}$.

\section{A. Excitation Method Comparison}

In an effort to verify that the excitation methods were not influencing the cable response in unexpected ways, a variety of excitation connections, methods, and signals were investigated. A random signal had to be used because the damped cable is a non-linear system and non-linear systems generate periodic noise. Signals for white noise and burst random from the shaker through the tensioned string were compared with hammer impacts, with the idea being that a similar response from the hammer impact would verify that the shaker connection was not affecting the response significantly. A Hanning window was applied for white noise signal and a rectangular window was applied for the burst random signal and hammer impacts. Hammer responses were tested with and without the load cell. As shown in Figure 5, the white noise and triggered burst random signals yielded nearly identical responses. The hammer impact responses bounded the string excitation responses, with the cable and load cell response showing a slightly lower natural frequency and the cable without the load cell attached showing a slightly higher frequency. It makes sense that added mass would result in a lower frequency, so modeling of the load cell must be taken into account for model comparison.

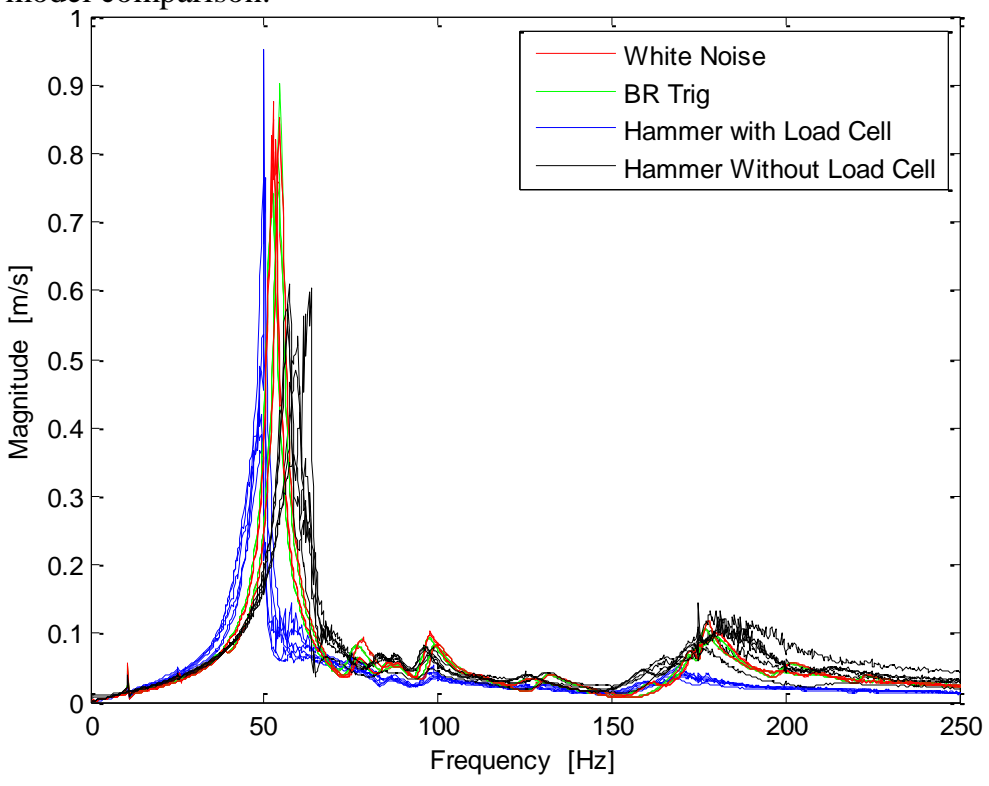

Figure 5. Frequency response functions of cable undergoing various excitation signals and methods.

\section{B. Excitation String Length and Tension Comparison}

To determine whether the excitation string was adding frequencies to the cable's frequency response, the excitation string length was tested at $4,10,17,24$, and 45 centimeters, with the DC offset kept constant and distance between cable fixture and shaker varied as the string length varied (thus ensuring constant tension in the excitation string). At $24 \mathrm{~cm}$ and $45 \mathrm{~cm}$, the DC offset was changed to measure the response for high, medium and low tension values, within the constraint of cable static bending less than 1 millimeter. Figure 6 shows the results from changes in string length for three representative days of testing; it is clear that string length is not affecting the cable response. Similarly, Figure 7 shows that the string tension is not a factor, although it should be stressed that this would not be the case if significant static bending were introduced due to the string tension. 

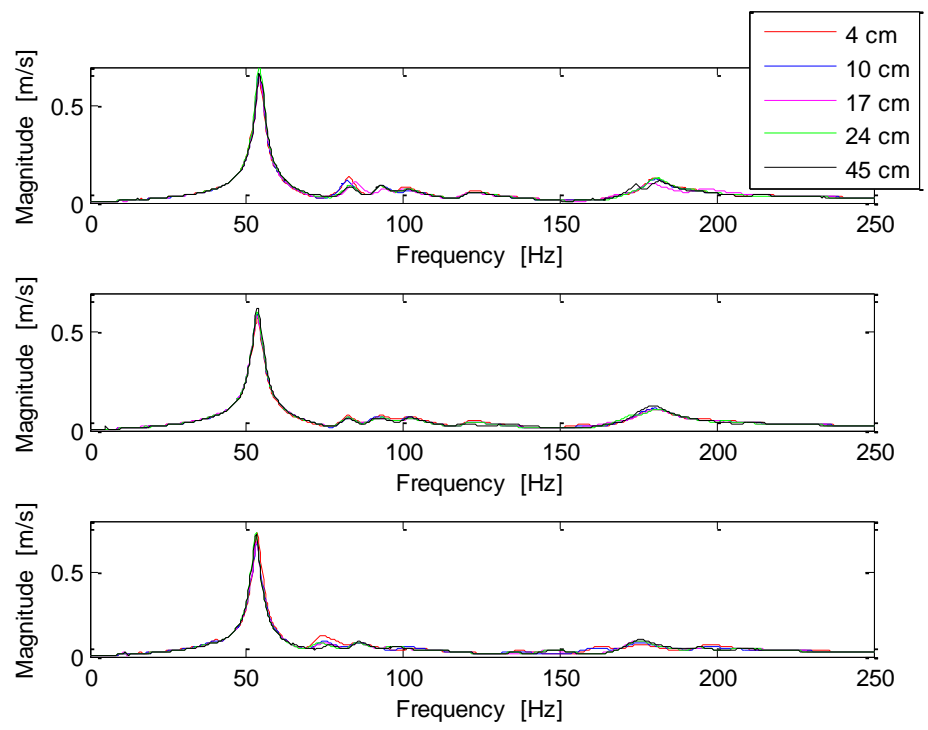

Figure 6. Comparison of excitation string length for three test days, showing lack of frequency dependence on string length
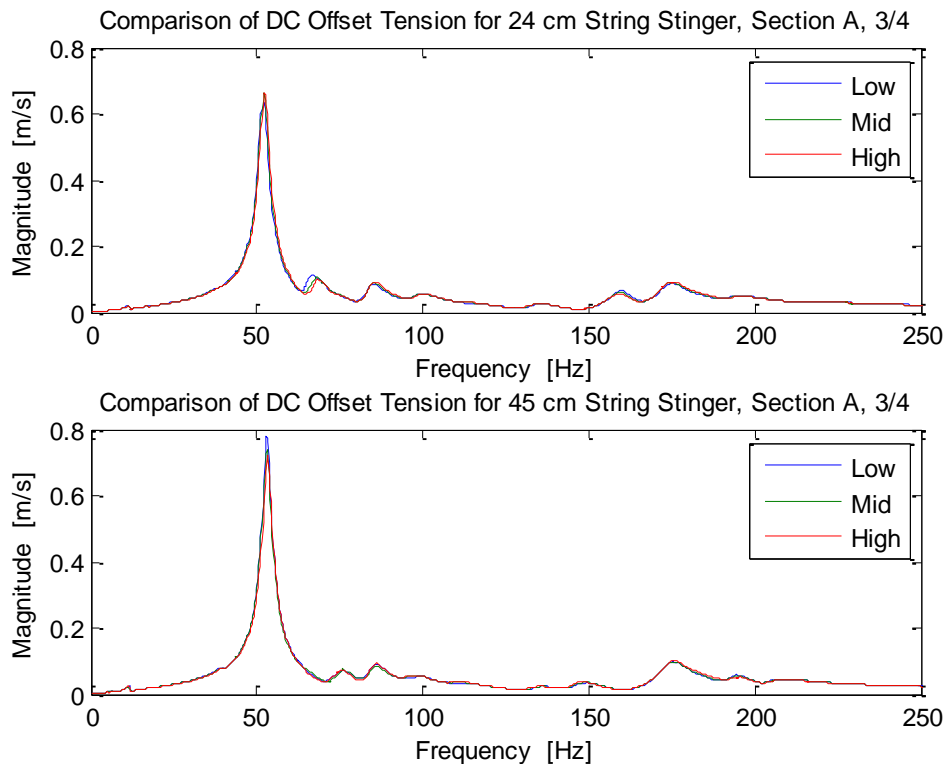

Figure 7. Comparison of tension in excitation string for 24 and $45 \mathrm{~cm}$ string lengths, showing lack of frequency dependence on tension for small static displacement of test cable

\section{Cable Tension Comparison}

It is well-known that strings exhibit higher frequencies at higher tensions, so it was expected that this same trend would be evident in cables. This test measured the cable response with tension in the cable of $1 \mathrm{lb}, 2 \mathrm{lb}, 3 \mathrm{lb}$, and 4 $\mathrm{lb}$, and also tested a slack cable and a "hand tight" cable, designed to simulate the tension a cable would experience if it was pulled snugly during space structure assembly. It turns out that "hand tight" fit within the testing parameters, which lends credibility to the use of these results for real world application. Figure 8 shows one of the test sets where the dissimilarities between the slack and tensioned cables are evident, especially around the second mode between 150 and $200 \mathrm{~Hz}$. Figure 9 enlarges the response near the first mode for three of the test sets to show that increasing tension does shift the frequency higher, though not as uniformly as a single wire (based on previous tests). Again, the cable pulled hand tight appears to give a similar response and the slack cable's first natural ferquency is lower than the others. 


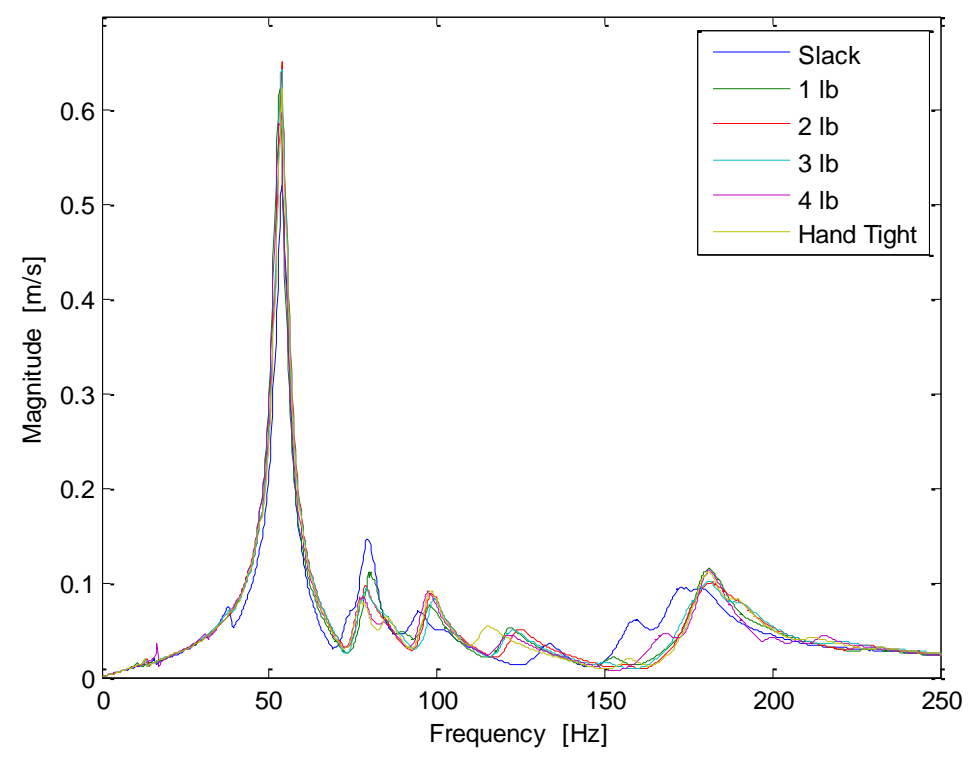

Figure 8. Representative cable tension test showing the difference between slack cable (blue line) and tensioned cables
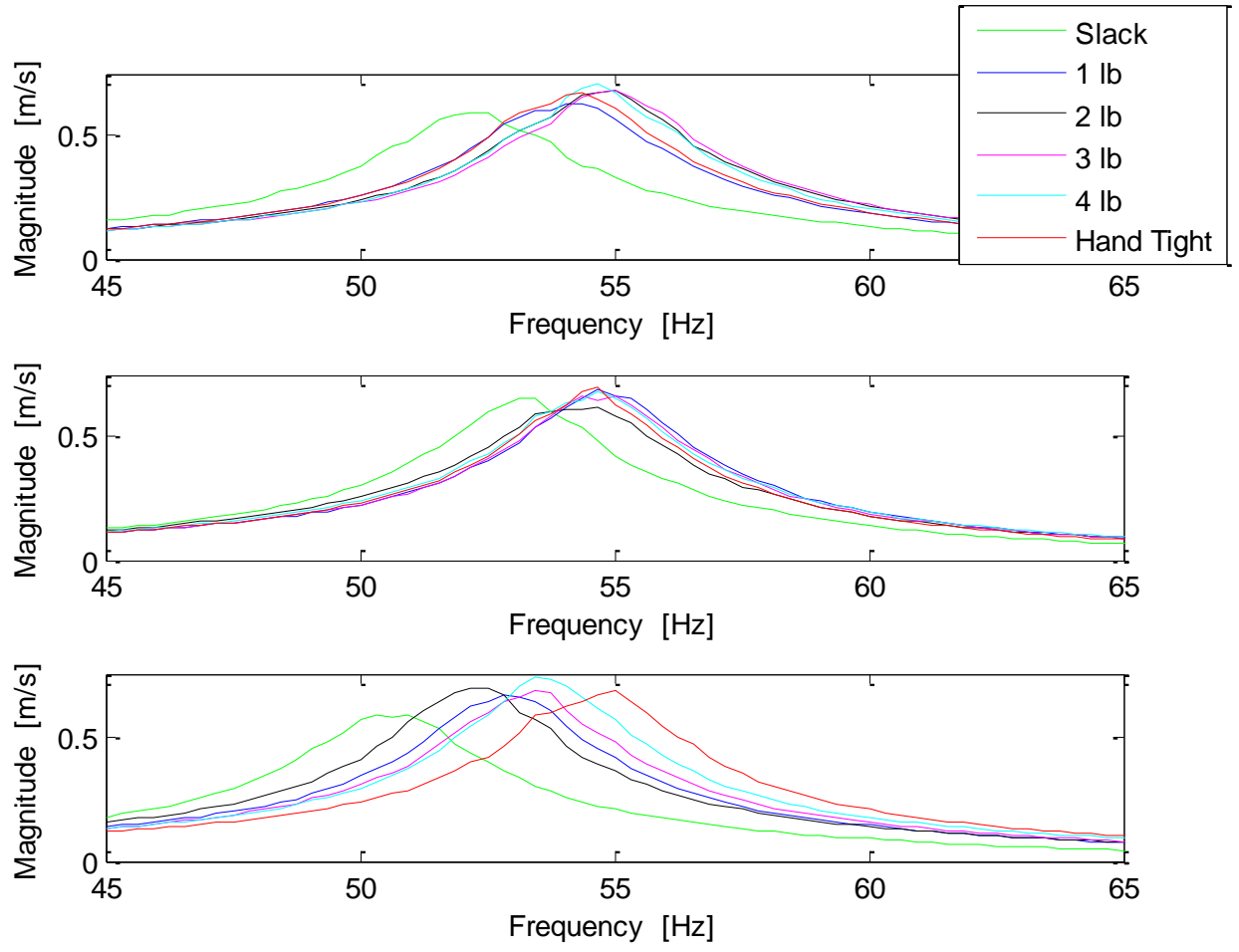

Figure 9. Cable response at first natural frequency showing the effect of cable tension on frequency for three test days

\section{Zip Tie Attachment Comparison}

Zip ties (also known as cable ties) are a common method for cable management in space structures. However, there is no real standard for how to fasten these cable management ties, so the effect of different types and tightness of zip ties were investigated. It turns out that the type or size of zip tie is not nearly as important as how tightly the zip tie is fastened. Zip tie guns can be adjusted to fasten zip ties to a specific tightness; for this test, "tight" implies setting 5 on a Thomas \& Betts Adjustable Tension Installing Tool WT-199 and "loose" implies setting 2. A "handloose" case was also tested, in which a zip tie was barely tightened enough to keep the cable from moving. This may simulate more of a pinned case as it allows the cable to pivot more. The hand loose case was significantly different, as shown by the red line in Fig. 10. Figure 10 shows the frequency responses for cables fastened with 
loose zip ties and cables fastened with tight zip ties; in contrast, Fig. 11 shows the responses from cables attached only with tightly fastened zip ties. The tighter zip tied cables yield more uniform and repeatable results, despite the fact that the ties varied in type and size.
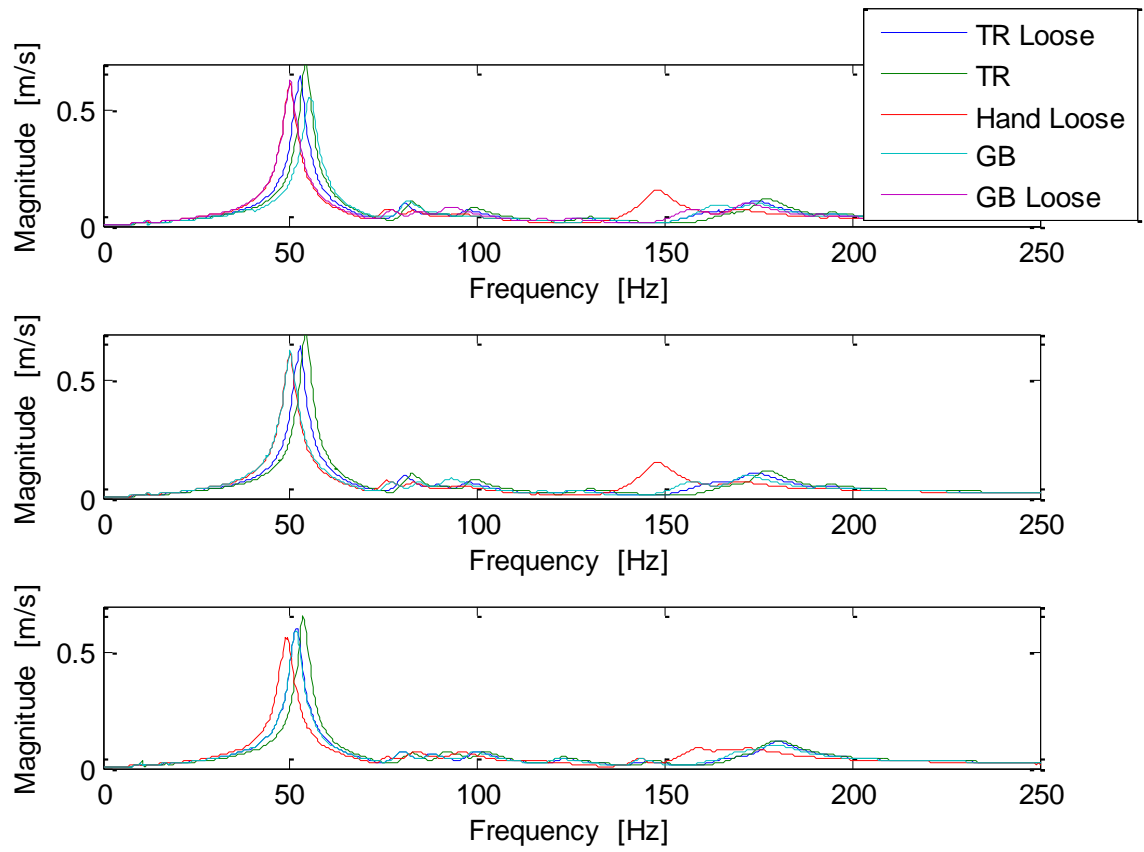

Figure 10. Comparison of loose and tight zip ties showing the variation due to zip tie tightness and the significant difference for handloose attachment (shown by red line)
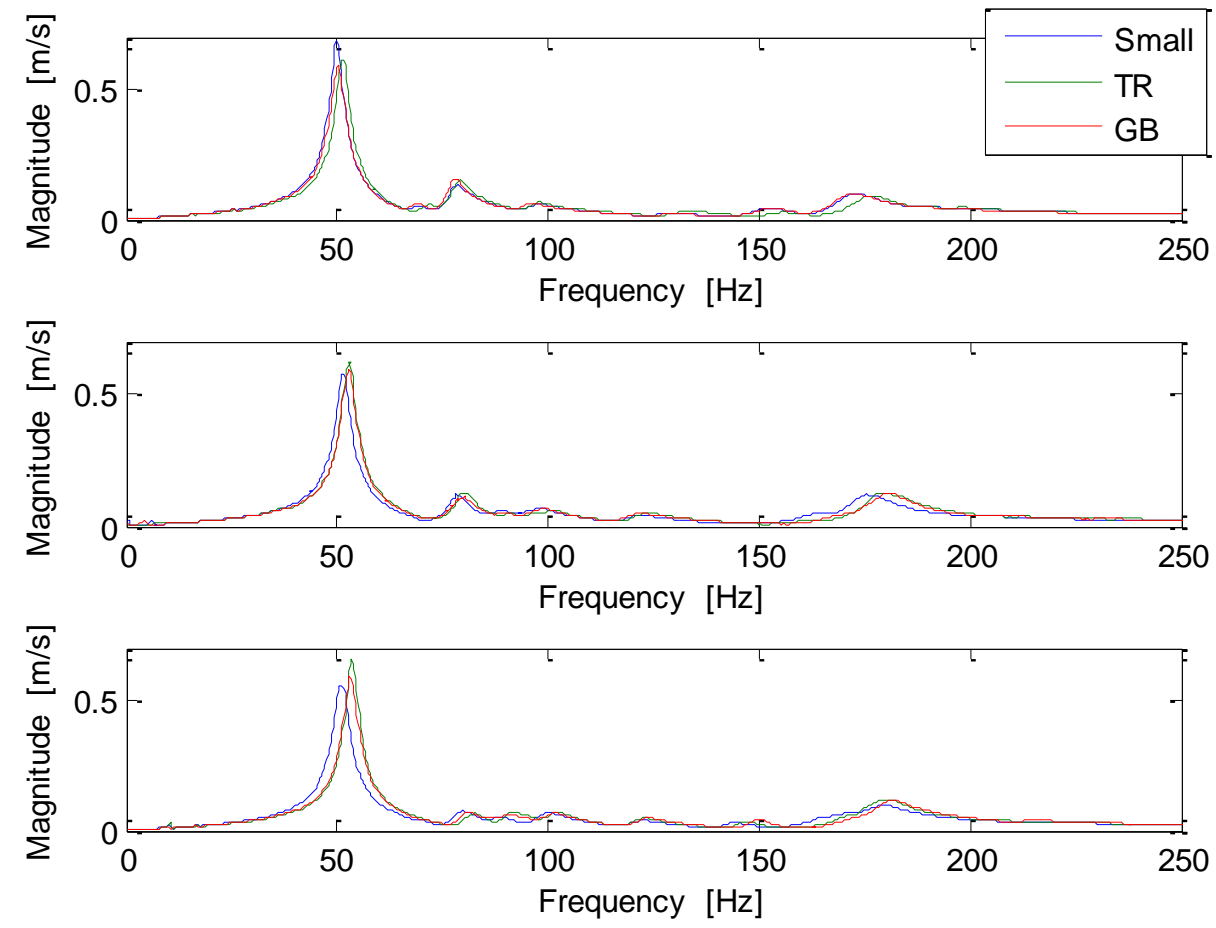

Figure 11. Comparison of tightly fastened zip ties of different size and type exhibiting the increased agreement between test runs as compared to loosely tightened zip ties

American Institute of Aeronautics and Astronautics 


\section{E. Effect of Cable Orientation in Test Fixture}

Although every effort was made during testing to orient the cables in the same way, with their natural coil toward the shaker, the authors decided to investigate just how much of an effect the cable orientation had on the frequency response. The tests show that the cable orientation in the test fixture was responsible for a large frequency range near the first natural frequency. In one test, illustrated in Fig. 12, the change in the first natural frequency was similar to the variation between cable sections as discussed in the next section. Figure 13 shows the shift in first natural frequency as compared to the angle of cable orientation in the test fixture; 0 degrees was intended to be the coil-plane parallel to the excitation with the coil facing toward the shaker, but shifting the 0 degree point for the cables showed greater similarity in the frequency-angle relationship shape. This is a good indication that the differences between cable sections shown in the next test may be due to different internal stresses in the cable; although all were aligned in the same way, different sections of cable may have varying internal stress tensors arising from their location in the overall cable and coiled storage. However, you can see from Fig. 13 that cables B and D still had higher frequencies throughout the tests, so .

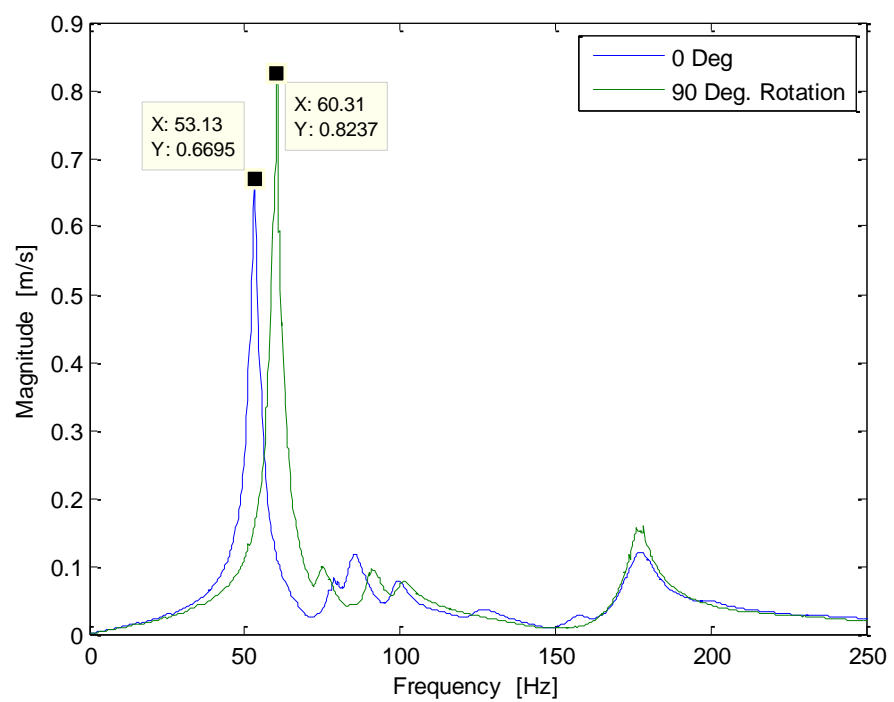

Figure 12. Frequency response function for a single cable at coil plane and 90 degree rotation from coil plane

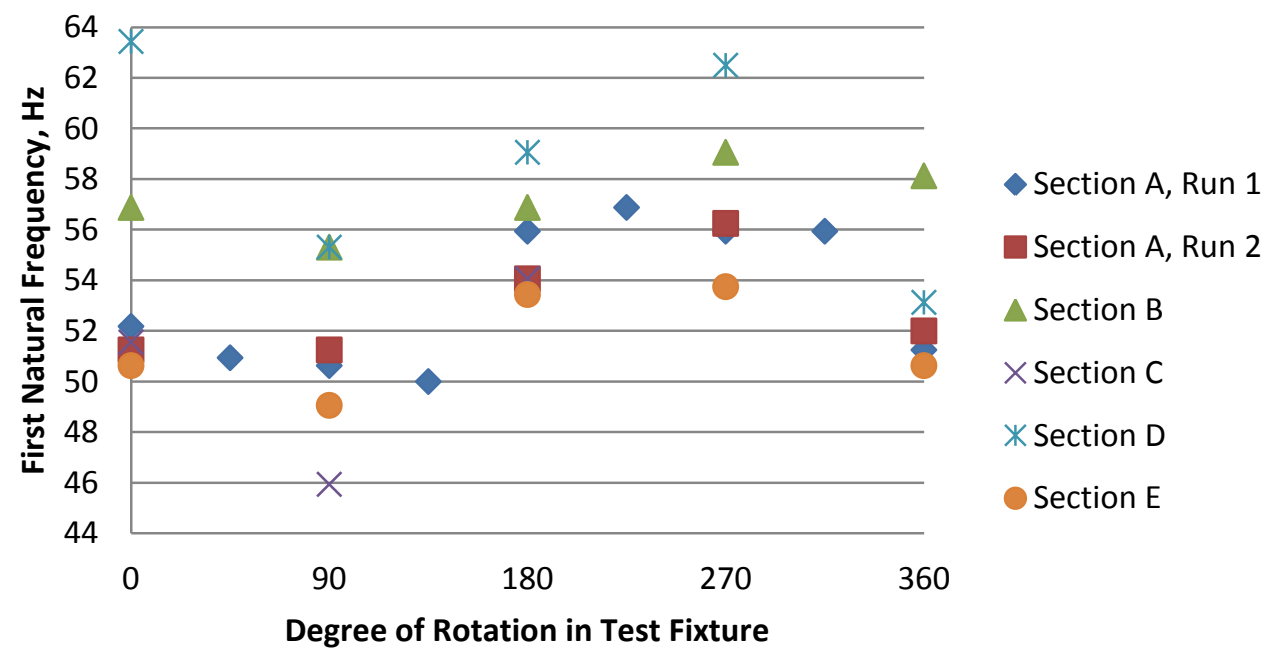

Figure 13. Approximately sinusoidal relationship between first natural frequency and cable fixture angle

\section{F. Comparison of Cable Sections}

As the preliminary tests were run, it was evident that the run-to-run variation even for a standard run of the same cable section was significant. Therefore, standard runs for the same cable section and different sections of the same 
cable were compared. Figure 14 shows the five cable sections, all from the same 150" length of cable, cut into five equal (and assumed identical!) sections. The next figures show the results, with a very clear separation between natural frequencies for the $\mathrm{A}, \mathrm{C}$ and $\mathrm{E}$ cables as a group and the $\mathrm{B}$ and $\mathrm{D}$ cables.
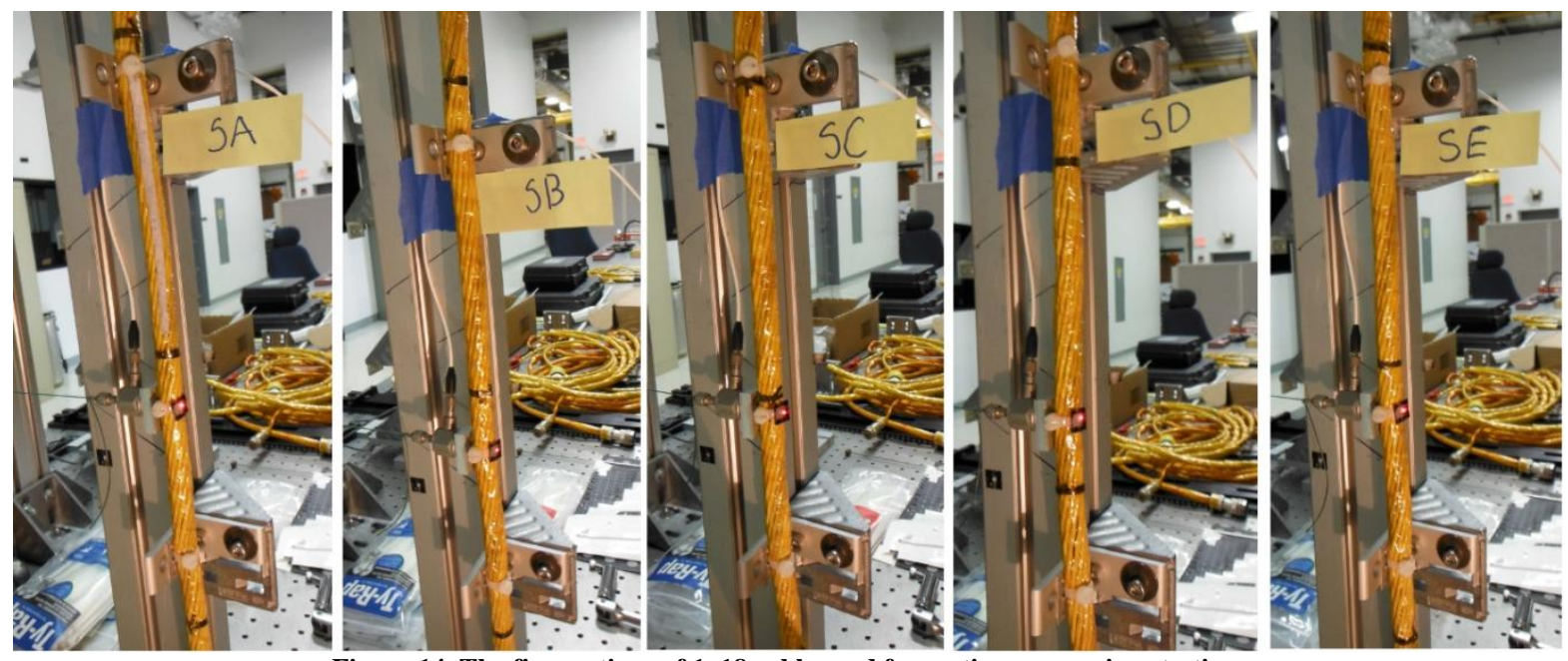

Figure 14. The five sections of $1 \times 18$ cable used for section comparison testing

Figure 15 shows the variation for a single section of cable (Section A, the section used for all variability tests). While the first and second natural frequencies appear in roughly the same place, there is slight variation in the frequency values and significant variation in the amount of damping (as measured by peak height). Thus, a statistical approach is likely to be necessary to determine the cable property parameters.

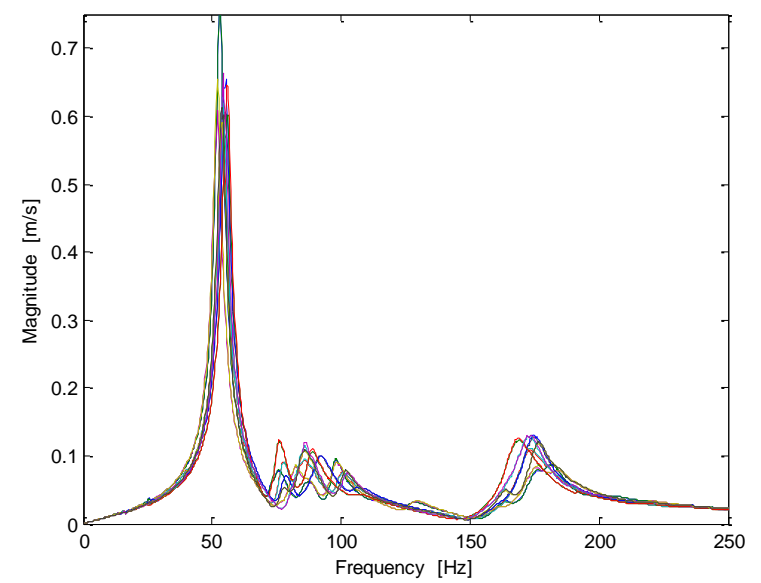

Figure 15. Frequency response functions for 14 standard runs of cable section A, showing the variation of response even for a single cable section

Figure 16 and Fig. 17 show the comparison between different sections of the same cable. As evidenced in Fig. 14, the only noticeable difference in these cable sections was the location of the black cable lacing ties relative to the driving point location. The sections were cut sequentially from a single 150 " piece of space flight suitable cable, and the Kapton overwrap and cable twist ties kept the helical cable structure intact. Lay angles were similar for all cable sections, and no visible differences were apparent. All cables were kept in a clamped test fixture overnight before first testing, and were stored hanging to prevent excessive curl from being stored coiled. The same amount of testing runs were performed on cables $\mathrm{B}, \mathrm{C}, \mathrm{D}$ and $\mathrm{E}$, with A tested more often due to its use in the variability testing. Overall, cables $\mathrm{A}$ and $\mathrm{D}$ showed the most similarity from run to run, and cable $\mathrm{C}$ showed the least. Figure 16 shows the agreement between sections $\mathrm{A}, \mathrm{C}$ and $\mathrm{E}$ and the agreement and frequency shift of sections B and D, where all runs of the same cable section are plotted in the same color. Figure 17 plots the runs of all cables together, clearly showing the two frequency peaks near the first mode and three frequency peaks near the second mode. All of these tests were run on the same day with the cable removed from the test set up and reattached between each run. 

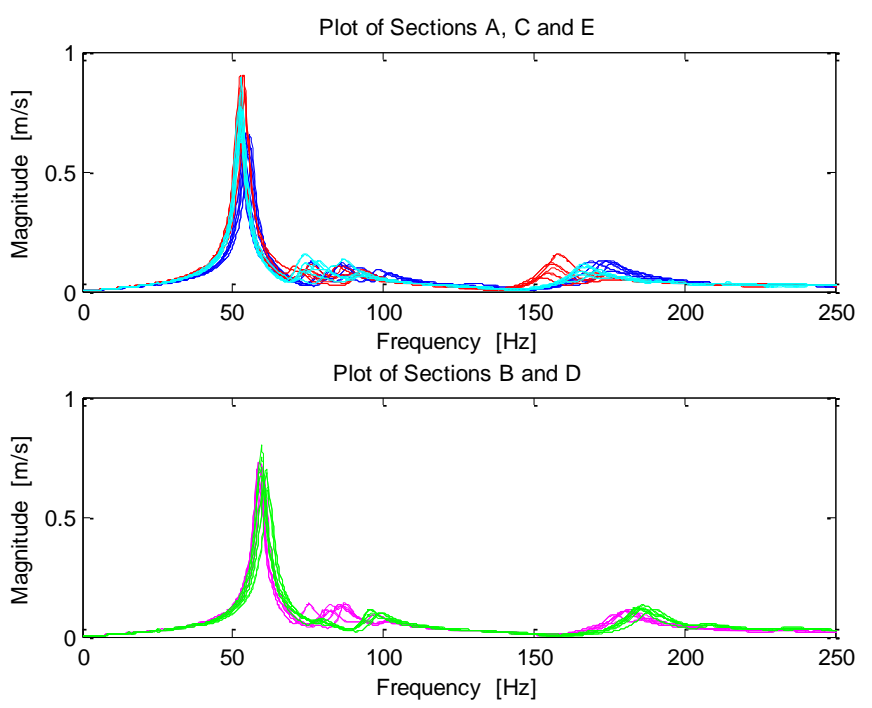

Figure 16. Frequency response functions for cable sections $A, C$ and $E$ and sections $B$ and $D$

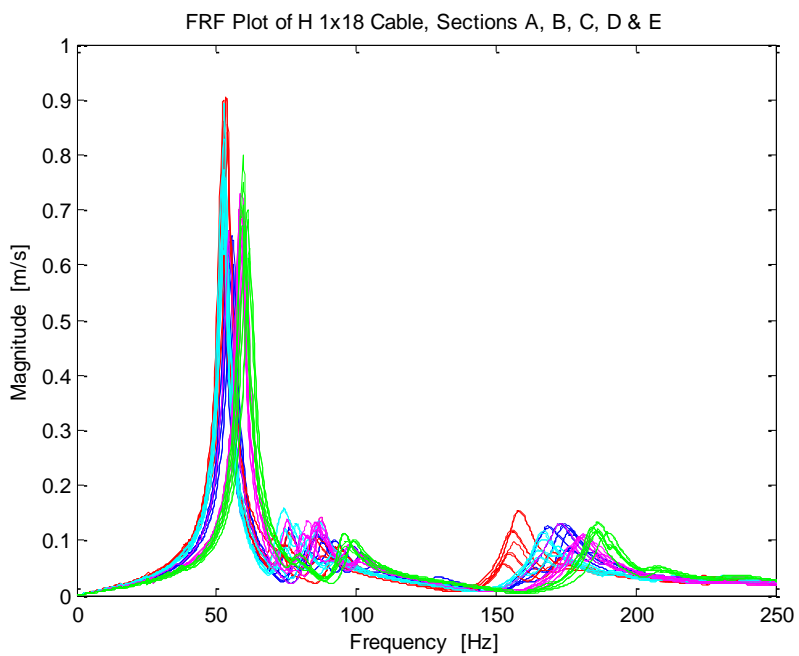

Figure 17. Frequency response functions for 10 runs of each cable section showing the dependence of frequency on cable section

\section{Discussion of Results}

The cable scans were valuable in verifying the first and second mode shapes and thus, natural frequencies. The trends for each test were fairly clear when analyzed at the conclusion of each day's testing, but when responses and frequencies were compared from day to day, there was great variation, even between "standard" runs. It was evident that tests which required the cable to be removed and then replaced in the test fixture had the greatest variation. Variation due to changes in the test set up were not significantly larger than variations between cable sections. The clear shift in natural frequencies for the B and D cable sections has not yet been satisfactorily explained, although a lack of dynamic symmetry in the cable may be the cause, as explained below. Further testing on these sections to determine their properties through dynamic mechanical analysis may help to determine whether the B and D cable sections actually have different dynamic properties, or if their orientation in the test set up explains the frequency shift.

An interesting result from the simultaneous measurement of the perpendicular vibrations was the observation that the symmetrical-appearing cable does not have the same natural frequencies in both directions. The cable is actually stiffer in one plane, which is not intuitive. It is hypothesized that the twisting of the cable, coiled storage and/or Kapton overwrap method may be responsible for this lack of symmetrical response. It is also worth noting that this could explain dual first frequencies shown between the two sets of cable sections; although every effort was made to orient the cables with curvature toward the shaker, if $\mathrm{B}$ and $\mathrm{D}$ repeatedly twisted as they were being installed, the stiffer plane would have been the primary measurement plane, which would explain the higher 
frequency. This could be a likely explanation; since the cables were stored in a coiled state before arriving at the test facility, they did have a tendancy to want to return to a specific shape, which did not necessarily coincide with the test shape. When section A was rotated 90 degrees, it's first natural frequency aligned with the B and D first natural frequency, around $60 \mathrm{~Hz}$. However, as shown in Fig. 13, B and D still had higher frequencies overall, which leads the authors to believe that there may be additional factors to be considered.

Conclusive results were obtained for the tests involving string length and tension, cable tension, and zip tie attachment. It was clear that for small cable deflections, the length of the excitation string and tension in the string were not affecting the cable's dynamic response. Cable tension did change the frequency response, with a general trend of higher tension corresponding to higher natural frequencies. This test also showed that "hand-tight" cables were on par with 1-4 lbs of tension in the cable, and that slack cables behave differently and may have more nonlinear attributes. Zip tie brand, type and size were not important factors, but the tightness of the zip tie attachment was. Therefore, cable tension and zip tie tightness should be controlled for future testing to reduce variation between standard runs. In addition, cable angle in the test fixture should be noted, as comparison between cables may require different orientations to test the same cable plane.

The excitation method of the cable went through several iterations, starting with a long solid stinger, hinged stingers, and eventually settling on the tensioned string used for these tests because of its ability to eliminate moments or lateral forces from being applied to the cable. The hammer tests bounded the tensioned string random excitation results, which seemed reasonable. The tests conducted yielded good representations of the cable dyanmics, with little interaction from the support structures.

The authors look forward to exploring the differences between cable sections and applying statistical methods to the cable section results to determine the cable parameters for model comparison.

\section{Future Work}

In the next phase of testing, several different cable constructions will be investigated; at the least, a $1 \mathrm{X} 7$ cable (one strand of seven wires), 1X 19 cable, (one strand of nineteen wires), 7 X 7 cable (seven strands of seven wires each, a double helix structure), and 1 X 49 cable (one strand of forty-nine wires) will be tested. This will give data for both first order helix structures (single strand cables) and second order helix structures (multi-strand cables). To the best of the authors' knowledge, this comparison has only been attempted through finite element models.

An overarching goal of compiling this experimental cable data is to use it to verify and validate theoretical cable models. Models are being developed using the distributed transfer function method (DTFM), a method well suited to eventually model cabled structures. Presented by Yang and Tan [5], the DTFM is an exact method that can be applied to both cables and cabled structures, and can handle a variety of additional factors such as tension, shear effects, viscous and hysteretic damping, and variable bending stiffness. To determine the cable parameters used in such a model, statistical analysis will be applied to the cable section data.

\section{Conclusion}

The authors hope that the experimental work presented here will provide a useful standard to compare theoretical cable damping models to, both for themselves and for other researchers in the field of cable dynamics. As well as expanding the availability of experimental cable data (needed, since some recent papers compare new theoretical models to experimental work performed 25 years ago, or rely only on FEM comparisons), a few of the factors that must be controlled for repeatable vibration testing have been identified. From this work, further progress has been made to investigate the damping of structures due to cable wiring harnesses.

\section{Acknowledgments}

The first author thanks the NASA Space Technology Research Fellowship program for generous support and the Virginia Space Grant Consortium for additional funding. The third author gratefully acknowledges the support of AFOSR Grant number FA9550-10-1-0427 monitored by Dr. David Stargel. Part of this research was carried out at the Jet Propulsion Laboratory, California Institute of Technology, under a contract with the National Aeronautics and Space Administration.

\section{References}

${ }^{1}$ Goodding, J. C., Ardelean, E. V., Babuska, V., Robertson, L. M., and Lane, S. A., "Experimental Techniques and Structural Parameter Estimation Studies of Spacecraft Cables," Journal of Spacecraft and Rockets, Vol. 48, No. 6, 2011, pp. 942-957. 
${ }^{2}$ Kauffman, J. L., Lesieutre, G. A., and Babuska, V., "Damping Models for Shear Beams with Applications to Spacecraft Wiring Harnesses," 53rd AIAA/ASME/ASCE/AHS/ASC Structures, Structural Dynamics and Materials Conference, Honolulu, HI, 2012, pp. 1-10.

${ }^{3}$ Friswell, M. I., Inman, D.J., and Lam, M. J., "On the Realization of GHM Models in Viscoelasticity," Journal of Intelligent Material Systems and Structures, Vol. 8, No. 11, 1997, pp. 986- 993.

${ }^{4}$ Banks H.T., and Inman D. J., "On Damping Mechanisms in Beams," Journal of Applied Mechanics, Vol. 58, Sept. 1991, pp 716-723.

${ }^{5}$ Yang B., and Tan C.A., "Transfer Functions of One-Dimensional Distributed Parameter Systems," Journal of Applied Mechanics, Vol. 59, Dec. 1992, pp. 1009-1014. 